\title{
A Hybrid Approach to Enhance Load Balancing in a Heterogeneous Cloud Environment
}

\author{
Boukari Souley $^{1}$, Abdullahi Nafisatu Aliyu ${ }^{2}$ \\ Department of Mathematical Science, Abubakar Tafawa Balewa University, Bauchi, Nigeria ${ }^{1,2}$
}

\begin{abstract}
Cloud computing is rapidly becoming more advanced .It has been put into use by very large set of users .It has become more attractive due to its cloud potentials of being easy to use and anywhere accessibility in comparison to other technologies. Load balancing is a very important component of efficient operations in cloud computing environment .Several algorithms have been designed but yet unable to rectify the holes found in the performance area. In this work we are proposing a hybrid approach to enhance performance for the cloud system user putting the concepts of enhanced Throttled and Equally Spread Current Execution algorithms (ESCE) into use. The proposed hybrid algorithm will be simulated in cloud analyst environment.
\end{abstract}

Keywords: Cloud Computing, Load Balancing, Performance, Cloud Analyst Network Delay, Physical Memory, Storage Capacity

\section{INTRODUCTION}

Cloud enables us to access information from anywhere at any giving time. Cloud computing is a type of computing concepts that involve a very large number of computers that are connected through a network such as internet. Cloud providers offers services as Infrastructure as a Services (IaaS), Platform as a Services (PaaS) and Software as a Services (SaaS). Cloud enable us to have access to information from anywhere at any time.[20].

Load balancing is a very important concept in network. The load balancer takes different requests from the client and distribute each of them across multiple computers or network devices depending on how busy the computer or network device is [7].

In a distributed system, the load balancing is a way of distributing the load among various nodes to make an improvement in active resource utilization and job response time. Load balancing is to assign the workload equally as soon as it is required. The traditional job scheduling algorithm makes impact on minimum number of jobs alone as static nature. But, this time around users need dynamic environment for obtaining fast job allocation and quick response time. In existing algorithms of load balancing there are many dynamic algorithms such as Ant Colony, Bee Colony, Bees Life algorithm, Particle Swarm Optimization and job scheduling algorithm.[20].

Load balancing algorithms for cloud computing distribute workload of multiple network links to achieve maximum throughput, minimize response time and to avoid overloading. Some of the algorithms were used to distribute the load and check the performance time and cost like the Round robin ESCE ,throttled etc. There are other works done using randomization such as ant colony algorithm [12].

The current load balancing scheduling algorithms in cloud computing environment such as Throttle ,Ant Colony, Equally Spread Current Execution Algorithm (ESCE) etc are not highly efficient. [5].With the ESCE load balancing algorithm by [2] , which has the advantages of maintaining equal load at all Virtual machines and Maximizing throughput, their work has a disadvantage of not tolerating fault. The throttled Load Balancing algorithm by [3] has the advantages of parsing Index table from the index next to already assigned virtual machine and faster response, their work has the disadvantages of not providing task queue at virtual machine level, virtual machines can only have one task at a time and they have no selection criteria for virtual machines. The Improved Throttled by [6] has the best advantage of maintaining the queue of task at virtual machine level, enabling the virtual machine to have more than one task and the selection of virtual machine is done base on priority of virtual machines.

[5] emphasize that hybridizing algorithms enhances the cloud computing performance. Several works had been done on hybridizing the algorithms for effective performance in the cloud like the work done by [16] etc.

Exploiting the advantages of both Improved throttled by [6], Equally Spread Current Execution (ESCE) algorithms by [2] can be a good frame work to improving the performance of cloud in terms of Response time, processing time, cost, physical memory and storage capacity. 


\section{REVIEW OF RELATED WORKS}

Some of the most important research works done on cloud computing emphasizing more on load balancing are being discussed in this section.

[1] proposed an optimized load balancing system for cloud using Particle Swarm Optimization (PSO) and Equally Spread Current Execution Load (ESCEL) algorithms as using both methods, system will be less complex and time will be reduce for client request as well as data centre request servicing time is also minimized. In the system cloud assign jobs or client request for jobs using ESCEL scheduling algorithm but before assigning jobs to VMs, these jobs were sent to the cloud server and cloud server optimized these jobs using Particle Swarm Optimization in an optimized way. Therefore, reducing the request time for client and data centre as well as fast response for completion of time is also delivered. In our proposed system client requests for resources to cloud server. These requests take a form of queue of jobs taking time as one of constraints. After queue formation of jobs, Particle Swarm Optimization (PSO) is applied for optimization of these tasks and then these tasks are sent to the cloud server as per computing purpose. Now, cloud server assigns these jobs to VMs after applying Equally Spread Current Execution Load (ESCEL) scheduling algorithm. As a result, Client gets optimized and fast response for completion of task is delivered. The drawback is that PSO was not optimized to make it appropriate for cloud environments and more efficient or effective in terms of load balancing.

[10] proposed work is to design an efficient scheduling algorithm that uniformly distribute workload among the available virtual machines in a data center and at the same time, decrease the overall response time and data center processing time. The proposed approach is a combination of Throttled and ESCE algorithm. Throttled algorithm makes use of states of VMs. A virtual machine state may be either AVAILABLE or BUSY. AVAILABLE state indicates that the virtual machine is idle/free and ready for cloudlet allotment, where BUSY state indicates that the current virtual machine is busy in execution of previous cloudlets and is not available to handle any new cloudlet request. This current load state of a VM helps in taking decision whether to allocate cloudlets to virtual machines or not. Active VM Load Balancing algorithms continuously monitor the job queue for new cloudlets and allot them to the bunch of idle/free VMs. It also maintains the list of cloudlets allocated to each virtual machine. This allocated cloudlet list helps in determining whether a VM is overloaded or under loaded at particular moment of time. On the basis of this information, VM load Balancer moves some load from overloaded VMs to the VM having minimum number of cloudlets, so as to maintain a high degree of balance among virtual machines. the drawback of this algorithm is that Overall data center processing time is not much improved in comparison to Equally Spread Current Execution Load( ESCE).

[16] worked on a Hybrid approach having concepts from Round Robin algorithm and Throttled algorithm proposed for virtual machine level load balancing. In the Proposed hybrid approach, initially when a cloudlet is received for execution on VMs then an arbitrary VM is chosen and checked for availability. If it is available then cloudlet is allocated to it. Otherwise control keeps moving in circular way through the VM list until it gets an available VM for cloudlet allocation. After allocation of cloudlet, next comparison takes place at the next VM coming in that circular way. the concept of circular way to allocate VMs to cloudlets has been taken from Round Robin algorithm and inspiration of checking availability on each step has been taken from throttled algorithm .The short coming is that Cost could not be decreased.

[5] proposed hybrid algorithm that takes advantages of both random and greedy algorithms; the algorithm adopts the characteristics of randomization and greedy to make an efficient load balancing and covers their disadvantages. The algorithm considers the current resource information and the CPU capacity factor to achieve the objectives. The hybrid algorithm consists of two main steps:

- In the first step VMs are distributed over hosts according to the host qualifications. The largest number of VMs are located at the most qualified host depending on the Hosts' CPU capacity.

- In the second step the algorithm used a new index table to record the current loads for each VM. And which used to check the current loads for VM at each iteration, the algorithm read the value of VM load from the index table; when the data center receives a request from the users, it sends the request to the hybrid load balancer. The hybrid algorithm will select $\mathrm{k}$ nodes (VM) randomly, and then it will choose the current load for each selected VM. Then it will choose a VM that have least VM current loads and return the VM id to Data center. The Data center will assign the load to the selected VM and update the value of selected VM in the index table of current loads. Finally when the VM finishes processing the request, it will inform the data center to updating its current load value. the limitation of this algorithm is that they tested only the effect of considering CPU capacity but there are other factors such as memory, bandwidth and storage. And other parameters for efficient utilization of resources such as cost, failover etc. were not considered.

[22] proposed an algorithm that combines the methodology of Divide-and-conquer and Throttled Algorithm (DCBT) which schedules the incoming requests to available VMs efficiently and ensures that there is no starvation of the requests. This hybrid approach consists of two algorithms: Pass I and Pass II. According to this algorithm requests from different clients are provided to the available Request Handlers (RH) and Virtual machines (VM). In the initial step, this algorithm checks for the availability of RH's and VM's and divides the requests accordingly using divide and 
conquer approach. In the next step, the incoming requests are assigned to the different RH's and VM's. Load Balancer keeps track of the current status of each RH or VM and verifies that the current request should only be assigned to the $\mathrm{RH}$ and VM which has not been used recently. This algorithm ensures that the load is distributed in an optimized way and no resource is idle thus leading to maximum resource utilization and minimum execution time thus leading to high performance. the drawback is that Deadline constraints are not considered.

[4] proposed a hybrid approach which combines (ACOPS) to solve scheduling in Virtual machines so that VM's are assigned to servers and resource usage is best utilized. ACOPS uses historical information to predict the workload of new input requests to adapt to dynamic environments without additional task information. Every time the request arrives, the initial step of ACOPS is Pre-reject in which the algorithm checks for the remaining memory of each server and will find the maximum amount of remaining memory. When the memory demands of request exceed the maximum remaining memory, the request will be rejected before scheduling. Search operator is used to construct the solutions for all ants. PSO operator is applied to improve the search result. In the next step, the evaluation operator is used to estimate the scheduling score of each ant. It will find the best solution and update the global best solution. In the next step, global pheromone updating is applied. This algorithm can serve requests for CPU, memory and disk utilization. Due to pre-reject step, computation time is reduced. PSO operator further reduces the computing time and improves the scheduling result. Thus, ACOPS ensures better load balancing than individual ant colony and particle swarm optimization algorithms. the drawback is that Although the algorithm can provide high load balancing, make span is shorter in single scheduling.

[23] proposed an Enhanced hybrid approach which is the advancement of hybrid algorithm that contains both Throttled and Equally Spread Current Execution algorithm. Enhanced Hybrid algorithm maintains an index list of VM allocation status as well as list to count the allocated request. The allocated request list is compared with the VMs index list. If VMs index list is greater than allocated request list it means that VMs are available to take request else request has been queued until VM is been available. If the VM has been queued, it has to wait in the queue itself. So new host has been created using host create function. In case of availability of VM, the jobs are allocated to that particular VM. And both the index list and hash list are updated. The job in queue needs not wait for long time for the virtual machine to become available. It Maximizes the resource utilization than the existing system. Minimize the response time and negligible idle time. it only analyzes the following parameters the overall response time, data processing time as well as data transfer cost .not putting other parameters into consideration.

[20] proposed system, where the ant colony and bees life algorithm are combined to improve the effectiveness of load balancing and cloud scheduling. The bees' life algorithm searches neighbor node and collect information (bandwidth, execution time, free memory space) from all neighbor nodes, and maintains as a table. If user gives any job as an input to cloud, the BLA first gets the job details and calculates the fitness for each job. From the fitness value, the best fitness value alone is taken to next generation. The BLA table information's are given to UN (Updating Node), this node also contains ANT COLONY table information such as past history of each node, available resource and best path to resource allocation. The UN node chooses the best path for resource allocation and shares the workload to all VMs. the short coming of the algorithm is that the resources utilization are balanced for only systems with multi resource constraints using hybrid $\mathrm{AB}$ colony algorithm.

[12] proposed composite algorithm is combination of two algorithms which are ESCE and throttled. The algorithm contains the advantages of both the algorithms. Firstly a Hash map is maintained which contains the number of virtual machines with their states(BUSY or AVAILABLE). Then throttled algorithm is used as when some request of client comes to data centre the load balancer scans the entire hash map list from top to bottom until first available virtual machine is found, if some virtually machine has state AVAILABLE then the request is allocated to that machine. If there is no virtual machine available then the request is queued. Now we utilize ESCE algorithm. In ESCE technique load balancer makes attempt to preserve equal load to all the virtual machines connected with the data center. In this technique load balancer gets number of virtual machines by maintaining an index table and queue of number of requests currently assigned to the virtual machine. If currently no virtual machine is available then Esce algorithm it looks for machine with minimum load and allocates the load to that virtual machine. the hybrid algorithm takes less processing time and response time. But the algorithm works good when no fault occurs in VM. The algorithm assumed the homogeneous VM not considering algorithm developed with heterogeneous VM.

27] proposed Hybrid Multiple Agent Genetic Algorithm for Load Balancing The main objective of the algorithm is to get a better results than traditional genetic algorithm. Genetic Algorithm is a search heuristic, based on evolutionary algorithm process of natural selection. GA mainly uses to repeat three steps - selection, crossover, and mutation . In MAGA, each individual MAGA is considered an agent, capable of sensing, changing, and impacting its environment autonomously, thus possessing its own characteristics. The genetic operators in case of MAGA include: neighborhood competition operator, neighborhood orthogonal crossover operator, mutation operator, and the self- learning operator. Among these operators, the neighborhood competition operator realized the operation of competition among all agents; the neighborhood orthogonal crossover operator achieved collaboration among agents; the mutation and self-learning operators accomplished the behavior that agents exploit their own knowledge . Results show that the hybrid algorithm is better than Min_min scheduling algorithm in terms of CPU utilization. It provides better and optimized results in 
handling high-dimensional function problems. This hybrid approach for achieving load balancing in cloud computing is thus more effective than Genetic Algorithm. The drawback is that To achieve higher performance in cloud, influence of memory will need to be ignored, thus providing load balancing for CPU only.

[14] proposed a system that established a new dynamic approach for effective load balancing used by Ant and Bee colony algorithm. A promote ant start travelling through the network .whenever this promote ant reaches a node, in this node is not the target node, it is heading for toward the destination. These promote ants have the same priority as data packet in node. When a promote ant reach the destination based on the provided in sequence by her, a toward the back bee is created then the promote ant is killed and new born bee continues the journey for next node. This toward the back bee traverse the promote ant's travelled route in reversed way and on its way update the pheromone values and is finally killed at the early node .the drawback of the algorithm is that they did not show the whole system in brief that provide good solution for effective load balancing using various QOS parameter in improved AB algorithm for better optimal solution.

[15] proposed a model to be used as a fault tolerance mechanism for real time computing on cloud infrastructure. It has all the advantages of forward recovery mechanism. It has a dynamic behavior of reliability configuration. The scheme is highly fault tolerant. This system takes the full advantage of using diverse software. In this experiment, they used three virtual machines. It utilizes all of three virtual machines in parallel. This scheme has incorporated the concept of fault tolerance on the basis of VM algorithm reliability. A Decision mechanism that shows convergence towards the result of the algorithm that has highest reliability. Probability of failure is very less in their devised scheme. This scheme works for forward recovery until all the nodes fail to produce the result. The system change the reliability by providing the backward recovery at two levels. First backward recovery point is Time Checker. Here if all the nodes fail to produce the result, it performs backward recovery. Second backward recovery point is Decision mechanism. It performs the backward recovery if the node with best reliability could not achieve the cc. It does not suffer from domino effect as check pointing is made in the end when all the nodes have produced the result. The reliable VM identification technique used in this process is very efficient to improve the QOS of cloud. They did not consider other enhancements to the model that can lead the system to be more fault-tolerant.

[6] proposed an approach, i.e. improved throttled approach, priority is assigned to each VM. Priority is calculated based on the capacity of VM and active allocated task count and size. The improved throttled scheduler will select that VM whose priority is highest among the available set of VM. A priority threshold level is also set to avoid overloading. If the priority of VM is less than priority threshold level, then the task is not allocated to that VM. Also, the scheduler will start searching VM in VM allocation table from the next to the last allocated VM. This will maintain the randomness in task VM mapping. The scheduler will maintain VM allocation table which will store VM id, VM capacity, Active task count, Status and Priority of VM. The drawback in this research is that they did not consider combining improved throttled with some other approach so that resultant becomes distributed in nature which, will result in a more suitable approach for cloud environments.

\section{CLOUD COMPUTING DEPLOYMENT MODELS}

Cloud deployments models as described by [13] are listed as follows:

\section{A. Public Cloud}

Public cloud this type of cloud is accessible by every single person using the internet. It is basically called the internet. It gives room for service providers to develop and deploy services so that users can have access and utilize services provided on pay. Public cloud is not that expensive and economical because all the cost of resource and services that are given such as application, hardware and network bandwidth are being taken care of by the provider. This type of cloud is usually not friendly to most organization because all the controls on this cloud are done by the third party and the privacy of sensitive data is not guarantee.

\section{B. Private Cloud}

Private clouds are developed and managed by an organization. This type of cloud is not sell-out to external users as it's developed mainly for use within the organization. Private cloud is more secured compared to public cloud since control of the resource is managed by the organization and privacy of sensitive data is maintained. Private cloud is very expensive to maintain because all the cost of the resource such as application, network bandwidth and hardware is incurred by the organization. Private cloud is usually not a good option for small and medium scale business organization but for large enterprises which have the ability to afford and maintain the resources.

\section{Hybrid Cloud}

Hybrid cloud is the coming together of public and private cloud. Hybrid cloud gives opportunity for organization or businesses to enjoy the services of both private and public cloud at the same time. In hybrid cloud, organization can manage their data in private and both in public base on demand. Meaning, critical sensitive data and information can be managed within the organization and less sensitive data can be uploaded to the public cloud. Hybrid cloud provide 
some essential benefits to the user since it can serve as backup in case there is a system failure or damage of data at one node due to natural disaster or unforeseen contingency.

D. Community Cloud

This type of cloud is been used by many organizations within the same community. This particular type of cloud is developed for the use of organizations that have the same privacy and security challenges such as banks and other important organizations to make them achieve their objectives. Community cloud can be managed without the interference of an external force by the organization or externally by a cloud service provider. The cost of the resources and services used is shared across the organizations within the community. Community cloud helps in minimizing cost and it is very good for joint ventures, research centers for developing and keeping their data in a centralized location.

\section{CLOUD SERVICE MODELS}

The following models can be used to deliver Cloud Computing services.

A. Infrastructure as a Service (IaaS)

This particular model of Cloud computing provide Hardware as a Service through Internet such as storage, CPU and many of them. There are other IaaS providers such as Amazon Elastic Cloud Compute (EC2), Rackspace e.t.c. [19].

B. Platform as a Service (PaaS)

Cloud computing gives a platform as a services that is needed for building application, where a particular user using tools and libraries for Cloud service providers, and also consumers deployed their applications without costing of hardware where providers of services provide the network, storage. There are various PaaS providers such as Google App Engine, Windows Azure e.t.c . [19].

C. Software as a Service (SaaS)

Deals with providing different software hosted on the Cloud and it is mostly referred to as on demand-software, where in this particular type of service, consumer will have to pay for His or Her use ofsoftware. Mostly consumer access to the software via the Internet, therefore, user uses the software where He /She does not require any integration with other system [19] There are several SaaS provider such as Google Apps, Sales Force. e.t.c [26].

\section{COMPONENTS IN CLOUD COMPUTING}

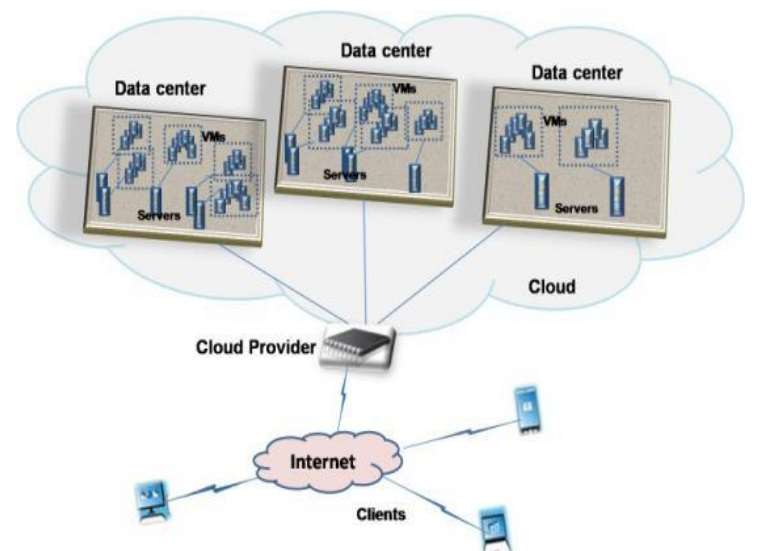

Fig. 1. Components in cloud computing.(Source: Hafiz etal 2015).

\section{CLOUD COMPUTING FEATURES}

Cloud computing gives many features that make it very recognized in the IT industry, such as Those listed below [26][19].

A. Absence of up-front investment: The cloud computing model of pricing is precisely based on pay-per-use principle. This model enables rent of services and resources from cloud as needed.

B. Lowering Cost Of Operation: Cloud environment resources are allocated and de-allocated on demand and this can give a recognized saving in operating costs since resources can be released whenever the service demand is low.

C. Scalability and Elasticity: The infrastructure providers have a vast amount of resources and infrastructure. So it is very easy for them to expand its service so as to handle the growing service. Based on the client demand [21]. In the 
other side, Elasticity is the ability to scale resources both up and down when required. Giving maximum opportunity to the dynamic integration and extraction of physical resources to the infrastructure. It is very clear that elasticity enables scalability [18].

D. Ease Of access: The cloud services are provided to users as a web-based services. So as to enable them get Access to the services through any of the devices supported with Internet connections.

E. Reducing business risks and maintenance expenses: Changes the business risks such as hardware failures to infrastructure providers, because providers have better expertise and resources to Take care of these risks [21].

F. Virtualization: Virtualization hides the characteristics of a computing platform's physical from its users [11], It gives room for abstraction and isolation of lower level functionalities and underlying hardware. [9]

G. Mobility: Cloud Computing means mobility because users can get access to applications through internet easily at all times. [17]

\section{LOAD BALANCING ALGORITHMS}

Load balancing algorithm deals with balancing the load on server nodes. The main responsibility of the load balancing algorithms is how to select next server node and to transfer new request to that particular node. Load balancing algorithms are classified as static and dynamic algorithms. [24] Static algorithms are mostly suitable for homogeneous and stable environments and can produce very good results in these environments. However, they are usually not flexible and cannot match the dynamic changes to the attributes during the execution time. Dynamic algorithms are more flexible and take into consideration different types of attributes in the system both prior to and during run-time.[8] These algorithms can adapt to changes and provide better performance results in heterogeneous and dynamic environments. However, as the distribution attributes become more complex and dynamic. As a result some of these algorithms could become inefficient and cause more overhead than necessary resulting in an overall degradation of the services [25].

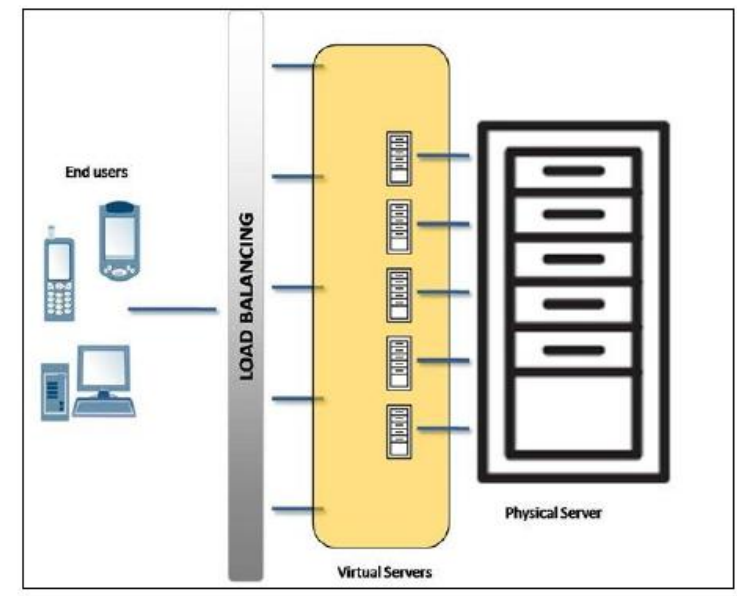

Fig. 2. Load Balancing in Cloud Computing.(source: Hafiz and Ala 2015).

As seen in the Figure 2 above, the data center needs a load balancing policy to process the request from users. The load balancer is responsible to assigns the virtual machine to the user request. Then the data center will send the response to the users after processing the Request. The load balancing is so important in cloud computing environment. The major goals of load balancing algorithms are:

- To achieve a whole improvement in system performance at a reasonable cost [17]

- To maintain a backup plan in a situation where the system fails even partially [29]

- To enable future modification in the system: the distributed system can change such as applying new topology and scale up. So a load balancing algorithm must have to be scalable and flexible to handle such changes.

\section{TYPES OF LOAD BALANCING ALGORITHMS}

- Static Load Balancing

In this type of load balancing algorithm the decision of shifting the load does not base solely on the current state of the system. It needs knowledge on the applications and resources of the system. The time at which job arrives determines the performance of the virtual machines. The master processor allocate the workload to other slave processors based on 
their performance. The allotted work is thus performed by the slave processors and the result is returned to the master processor.

- Dynamic Load Balancing

This type of load balancing algorithms make use of the current state of the system to make any kind of decision for load balancing, the current state of the system also takes control of the shifting of the load. It allows for processes to move from an over utilized machine to an under-utilized machine dynamically for faster execution. this approach important because its decision for balancing the load is based on the current state of the system which helps in improving the overall performance of the system by migrating the load dynamically. [28]

\section{EXISTING LOAD BALANCING ALGORITHMS}

A. Equally Spread Current Execution Algorithm (ESCE): By [2]. The load balancer tries to preserve equal load to all the virtual machines connected with the data centre. In Equally spread current execution algorithm, the processes are handled with load priorities. It distributes the load to virtual machine by checking the load at current time and transfer of the load to that virtual machine which is lightly loaded and handles that request easily and result in less time taken , and give maximum possible throughput It is spread spectrum technique in which the load balancer spreads the load into multiple virtual machines.

B. Throttled Load Balancing Algorithm (TLB): By [3] In TLB algorithm, an index table is maintained by load balancer which contains virtual machines as well as their states (Available or Busy). On receiving a request from client data centre firstly tries to find a suitable virtual machine (VM) to perform the requested task. The data centre broker queries the load balancer for allocation of the VM. The load balancer scans the index table from top until the first available VM is found or the index table is scanned fully. If the status of any VM is Available, then VM id is send to the data centre. The data centre then allocates the request to the VM identified using the throttled algorithm. Also, the data centre updates the index table and set the state of $\mathrm{Vm}$ to busy. But during processing the request of client, if no VM is found, the load balancer returns -1 to the data centre .

C. Improved Throttled Algorithm: The improved throttled Algorithm by [6] is devoted to performance enhancement for the user of the existing cloud system by improving the basic throttled mapping approach between task and resources. the improved throttled algorithm was achieved through the use of priority. by setting priority to each VM. The Priority is calculated based on the capacity of VM and active allocated task count and size. The improved throttled scheduler will select that VM whose priority is highest among the available set of VM. A priority threshold level is also set to avoid overloading. If the priority of VM is less than priority threshold level, then the task is not allocated to that VM. Also, the scheduler will start searching VM in VM allocation table from the next to the last allocated VM. This will maintain the randomness in task VM mapping. The scheduler will maintain VM allocation table which will store VM id, VM capacity, Active task count, Status and Priority of VM.

\section{METHODOLOGY}

This research proposes a hybrid approach to enhance scheduling algorithms that will take the advantages of the equally Spread Current Execution (ESCE) by [2] and Improved Throttled Algorithms by [6]. The algorithm will consider the physical memory with dynamic processor power and storage capacity in a heterogeneous environment to achieve sufficient efficiency. The first step is to design a hybrid algorithm based on Equally spread current execution (ESCE) and Improved Throttled Algorithm, then test the hybrid load balancing algorithm's performance in a heterogeneous environment of hosts, then study the problem with the effect of network delay, then test the effect of considering the physical memory on the hybrid algorithm, then test the effect of storage capacity on the hybrid algorithm . Finally will compare the hybrid algorithm with existing ESCE by [2] and the improved throttled by [6] to obtain results.

\section{PROPOSED SYSTEM}

In this work a hybrid load balancing algorithm based on Equally Spread Current Execution Algorithm ESCE by [2] which will equally distribute loads to virtual machines and Improved Throttled Algorithms by [6] will be developed, the distribution of loads to virtual machines will be done according to VM's priority. The following steps below will be followed to achieve the work.

- The proposed Algorithm based on improved throttled and equally spread current execution Algorithm will be designed.

- Use cloud analyst to implement the proposed algorithm.

- The proposed algorithm will be tested in a heterogeneous of CPU and physical memory with the effect of network delay. 
- The proposed algorithm will test in a heterogeneous of CPU and physical memory considering the effect of physical memory and storage capacity.

The proposed algorithm will test the effect of Network delay, storage capacity and physical memory in a heterogeneous of CPU and memory.

\section{WORKING PRINCIPLE OF THE PROPOSED SYSTEM}

This Research will develop a Hybrid Algorithm to obtain better performance in load balancing based on Improved Throttled Algorithm by ]6] and Equally Spread Current Execution Algorithm by [2]which will lead to achieving better response Time, Processing Time and cost by testing the effect of Network Delay, Physical Memory and Storage Capacity. The performance of the proposed Algorithm will be evaluated against the existing improved throttled by [6] and Equally Spread Current Execution (ESCE) by [2] using cloud analyst simulator making emphasis on dynamic number of CPU and varied Physical Memory.

\section{CONCEPTUAL FRAME WORK OF THE PROPOSED SYSTEM}

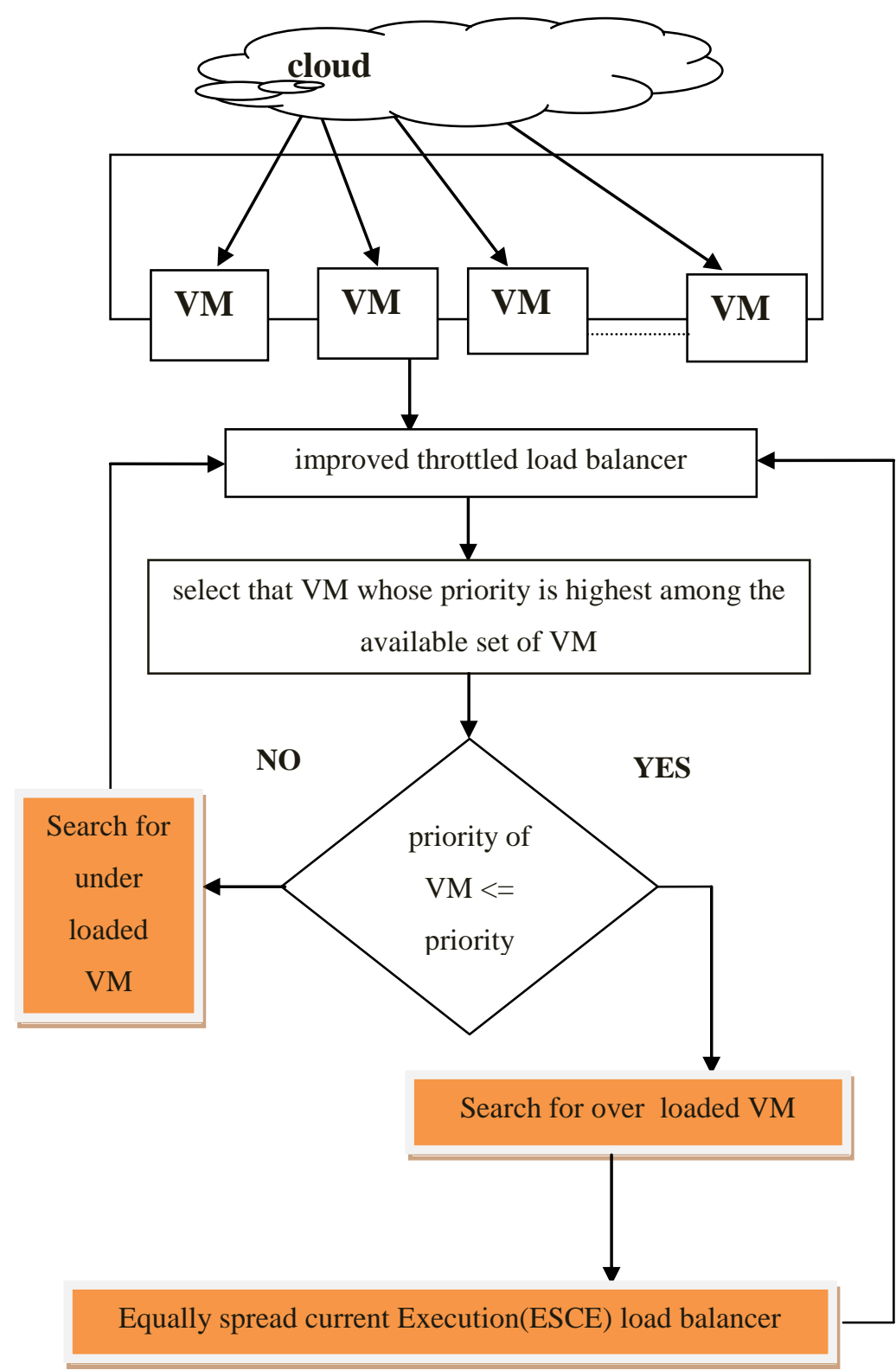

Figure 3:Architecture of the proposed system 


\section{CONCLUTION AND FUTURE WORK}

In the next work the hybrid model combining improved Throttled and Equally Spread Current Execution (ESCE) algorithm will be evaluated based on Network delay, Physical memory and storage capacity with dynamic number of processors and physical memory in a heterogeneous cloud environment. The proposed Hybrid algorithm is expected to provide better response time, Processing time and Grand total cost and it is also expected to be better in maximum times than the improved throttled by [6] and the Equally Spread Current Execution Algorithm ESCE by [2]. In the current work we will recommend that in future, fault tolerance and other performances such as Bandwidth, usability etc are to be analyzed so as to ensure a better performance in load balancing and cloud computing in general.

\section{REFERENCES}

[1]. Anoop, Y.(2015) Comparative Analysis of Load Balancing Algorithms in Cloud Computing International Journal of Enhanced Research in Management \& Computer Applications. Department of Computer Science and Engineering,4(9),61-67.

[2]. Bhathiya, W. (2010) - Cloud Analyst: A Cloud-Sim-Based Tool For Modeling And Analysis Of Large Scale Cloud Computing Environments. MEDC Projectll, Report.

[3]. Bhathiya, W. and Roderigo, N.(2010). Cloud Analyst: A Cloud-Sim-Based Visual Modeler For Analyzing Cloud Computing Environments And Applications. Proc Of IEEE International Conference On Advance Information Networking And Applications.

[4]. Cho K., Tsai P., Tsai C. ,(2014) A hybrid meta-heuristic algorithm for VM scheduling with load balancing in cloud computing Neural Computing and Applications. 26(6),1-13.

[5]. Hafiz, J.\& Alaa E.(2015) Efficient Load Balancing Algorithm in Cloud Computing. International Journal of Soft Computing and Engineering , 5(3) 2231-2307.

[6]. Imtiyaz, A., shakeel, A. and souurav, .M (2017) An enhanced load balancing throttled approach for cloud environment International Research Journal of Engineering and Technology.4(6),512-517.

[7]. Kalaithem, S., Mary, L. and Immaculate, S. (2014) A Survey of Load Balancing Algorithms using VM's International Journal of Advancements in Research \& Technology, SciResPub.3(8),68-76.

[8]. Klaithem, A., Nader, M., Mariam A., and Jameela, A.(2014) A Novel Approach for Dual-Direction Load Balancing and Storage Optimization in Cloud Services ,IEEE 13th International Symposium on Network Computing and Applications.

[9]. Kumar, S., and Aramudhan, M.(2013) Performance Analysis of Cloud under different Virtual Machine Capacity International Journal of Computer Applications, 68(8), 1-4.

[10]. Mamta, K. \& Sanjay, T. (2016). An efficient algorithm for load balancing in cloud computing. International Journal Of Engineering Sciences \& Research Technology. 2277-9655

[11]. Marisol, G., Cucinotta, T. and Lu, C.(2014).Challenges in real-time virtualization and predictable cloud computing. Journal of Systems Architecture (ELSEVIER), 1-15.

[12]. Navtej, S. Rajesh, S. (2016). An Efficient Approach For Load Balancing In Cloud Computing Using Composite Techniques International Journal of Research in Engineering and Applied Sciences.6(2),145-149.

[13]. Neha, M. K. (2016). Enhanced Security using Hybrid Encryption Algorithm. International Journal of Innovative Research in Computer and Communication Engineering, 4(7), 13001-13007.

[14]. Pooja B. \& Piyush, V.(2015). An Effective Load Balancing Approach In Cloud Using Dynamic AB Algorithm International Journal Of Innovative Research In Science, Engineering And Technology.4(8),7389-7396.

[15]. Rajesh, S. and Kanniga, D.(2014). Improving Fault Tolerance in Virtual Machine Based Cloud Infrastructure.International Conference on Innovations in Engineering and Technology.

[16]. Rajkumar, S. \& Jyotsana O.(2014). A Hybrid Approach for VM Load Balancing in Cloud Using CloudSim International Journal of Science, Engineering and Technology Research.3(6) ,1734-1739.

[17]. Ray, S. and De Sarkar, A.(2012).Execution analysis of load balancing algorithms in cloud computing environment. International Journal on Cloud Computing: Services and Architecture (IJCCSA), 2(5),1-13.

[18]. Sajid, M. \& Raza, Z.(2013). Cloud Computing: Issues \& Challenges. in International Conference on Cloud. 35-41.

[19]. Sareen, P.(2013). Cloud Computing: Types, Architecture, Applications, Concerns, Virtualization and Role of IT Governance in Cloud. International Journal of Advanced Research in Computer Science and Software Engineering, 3(3), 533-538.

[20]. Sasikala N. and Ramesh D. (2014). Effective Load Balancing for Cloud Computing using Hybrid AB Algorithm. international journal of innovative research in computer and communication engineering,2(4), 3815-3822.

[21]. Shah, J.(2014) Cloud Computing: The Technology for Next Generation. International Journal of Advances in Computer Science and Technology, 3(3), 152-155.

[22]. Shridhar G. and Ram M.,(2013) Load Balancing in Cloud Computing Using Modified Throttled Algorithm ,IEEE International Conference on Cloud Computing in Emerging Markets (CCEM).

[23]. Subalakshmi, S. \& Malarvizhi, N. (2017). Enhanced Hybrid Approach for Load Balancing Algorithms in Cloud Computing' International Journal of Scientific Research in Computer Science, Engineering and Information Technology.2(2),136-142

[24]. Vasudha A. \& Tyagi S., (2014).Performance Evaluation of Load Balancing Policies across Virtual Machines in a Data Center, International Conference on Reliability, Optimization and Information Technology.

[25]. Velagapudi, S., Prathap. M, \& Mohammed K.(2009). Load Balancing Techniques: Major Challenge in Cloud Computing - A Systematic Review'. Proceedings of 5th IEEE International Joint Conference on INC, IMS and IDC, Seoul, Korea,

[26]. Zhang, Q., Cheng, L., and Boutaba, R.,(2010) Cloud computing: state-of-the-art and research challenges. Journal of Internet Services and Applications, 1(1), 7-18.

[27]. Zhu, K., Huaguang S., Lijing L., Jinzhu G.\& Guojian C.(2011). Hybrid Genetic Algorithm for Cloud Computing Applications, IEEE International Conference on Asia-Pacific Services Computing Conference (APSCC).

[28]. Radha R. (2015). An Approach to Load Balancing In Cloud Computing, Copyright to IJIRSET 4(5), 3769-3777.

[29]. Padhy, R.P. \& Rao, G.P. (2011) Load Balancing in Cloud Computing Systems: Orissa, India. 